\title{
Radical Reactions of Boron-Ate Complexes Promoting a 1,2-Metallate Rearrangement
}

\author{
Nicholas D. C. Tappin and Philippe Renaud ${ }^{*}$
}

\begin{abstract}
Recently there has been an explosion of interest in the synthetic community for the addition of radicals into unsaturated organoboron-ate complexes. This review will give a concise outline for radical processes involving boron-ate complexes which trigger a subsequent anionotropic rearrangement.
\end{abstract}

Keywords: Boronic esters · Boron-ate complexes · Cross-coupling · Inverse ylid · 1,2-Metallate rearrangement $\cdot$ Matteson rearrangement $\cdot$ Radicals

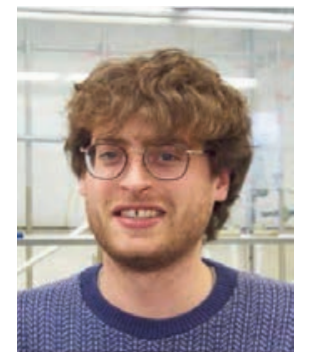

Nicholas D. C. Tappin received an MSci in Physical Natural Sciences in 2009 from Queens' College, University of Cambridge, with a research project in the Innovative Technology Centre of the Ley Group on the flow synthesis of PyBox ligands. After sojourns at the University of California, Santa Barbara, under Prof. Zakarian, and the Ecole polytechnique fédérale de Lausanne, under Prof. Zhu, he finally settled on the subject of 1-boryl radicals at the University of Bern to complete his PhD in 2019 under the supervision of Prof. Renaud.

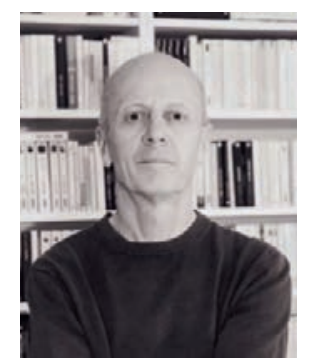

Philippe Renaud was born in Neuchâtel. After undergraduate studies at the University of Neuchâtel, he continued his education at the ETH Zürich with the PhD in 1986 under the supervision of Prof. D. Seebach followed by a postdoc with Prof. M.A. Fox at the University of Texas at Austin. In 1988, he started an independent research program at the University of Lausanne. In October 1993, he moved to the University of Fribourg as an associate professor. Since March 2001, he is professor of organic chemistry at the University of Bern.

\section{Introduction}

Chemistry involving organoboron compounds has been of long-standing interest to synthetic chemists. The rich and diverse chemistry of organoboranes and the low toxicity of boron derivatives make them particularly attractive in the context of increasing sustainability of synthetic methods by replacing toxic and hazardous reagents. Beside the classical Suzuki-Miyaura and related cross-coupling reactions, ${ }^{[1,2]}$ radical chemistry involving organoboranes has expanded dramatically over the past years. ${ }^{[3-6]}$ Recently, spectacular developments have been achieved in the radical addition to unsaturated boronic ester derivatives making use of the boron atom's unique properties (it is either metal- lic or non-metallic depending on its ligand sphere). Beside the standard radical addition to unsaturated 1-alkenylboronic esters (Scheme 1, A), addition to complexed boronic esters (Scheme 1, B) and to 1-alkenylboronates (Scheme 1, C) have been reported. The first two processes (Scheme 1, A and B) occur in conjunction with classical radical chemistry such as tin hydride-mediated reactions, ${ }^{[7]}$ photoredox catalyzed processes ${ }^{[8]}$ and atom/group transfer chemistry. ${ }^{[9]}$ The third approach (Scheme 1, C) affords an unusual electron-rich radical anion species that can act as a strong one-electron reducing agent, triggering a 1,2-metallate rearrangement (Matteson-type rearrangement) on itself. Related ionic transition-metal catalyzed processes have been reported and recently reviewed. ${ }^{[10]}$

Our review will start briefly with a discussion of simple atom and group transfer radical addition to complexed 1-alkenylboronic acid derivatives and 1-alkenylboronates and will then focus on radical addition to 1-alkenylboronates resulting in 1,2-metallate rearrangements. This new class of radical-polar crossover reactions represents a unique entry into three-component coupling reactions between a nucleophile (mostly organolithium), an electrophilic halide, and an alkene.

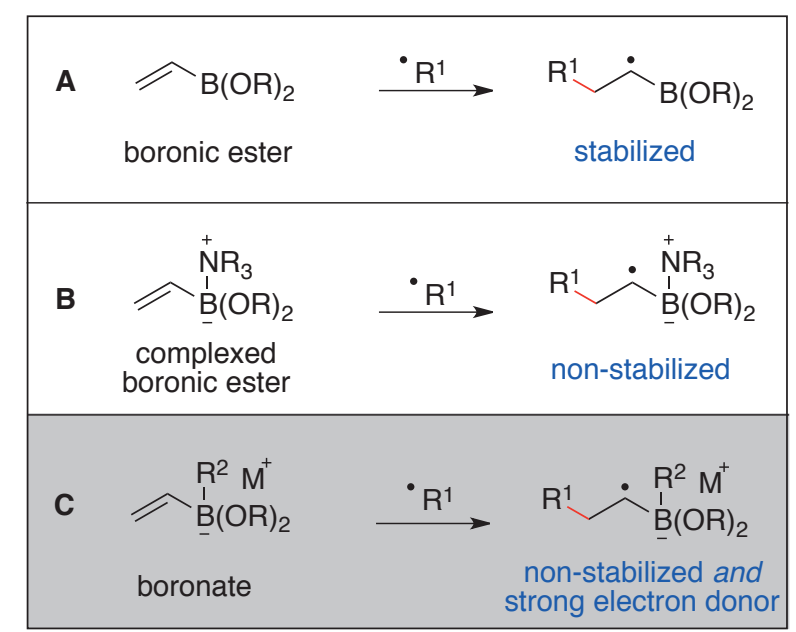

Scheme 1. Radical addition to unsaturated boronic ester derivatives. 


$$
\text { (SC(M) }
$$

Scheme 2. Xanthate addition over MIDA vinylboronates.

\section{Simple Radical Addition to Complexed Boronic Esters and Unsaturated Boronates}

Zard and co-workers took advantage of the inability of the complexed $\mathrm{sp}^{3}$-hybridized boron atom in $\mathrm{N}$-methyliminodiacetyl (MIDA) boronic acid derivatives to stabilize an adjacent radical[11,12] to develop an efficient addition of radicals to MIDA vinylboronates under xanthate transfer conditions initiated by dilauroyl peroxide (DLP) ${ }^{[9]}$ In contrast to vinylboronic pinacol esters, ${ }^{[13,14]}$ the MIDA vinylboronates behave as electron-rich alkenes affording good yields with electron-deficient radicals (Scheme 2).

In a related work, Kublicki and co-workers reported the iodine atom transfer radical addition to MIDA vinyl- and alkynylborates under photoredox initiation (Scheme 3). ${ }^{[15]}$

The reaction was extended to 1-alkenyltrifluoroborates (Scheme 4).[16] Again using photoredox initiation, they were able to run iodine atom transfer addition also with perfluoroalkyl radicals.

Radical additions at position 1 of 1-alkenylboronates leading intermediate 2-boryl radicals are also known and have served as the basis for the alkenylation of radicals.[17-20]

\section{Discovery of the Three-component Coupling Reactions}

Matteson added carbon tetrachloride over dibutyl vinylboronates in $45 \%$ yield by simple initiation with AIBN, and in excellent yields with bromotrichloromethane. ${ }^{[21,22]}$ Upon treatment of the intermediate 1-bromoalkaneboronic ester with aryl Grignard reagents, Matteson discovered the now eponymous 1,2-metallate rearrangement (Scheme 5). ${ }^{[23-25]}$

Recently, a number of research groups have reported a threecomponent coupling reaction that telescopes Matteson's chemistry into a one-pot process by reversing the order of the ate formation and radical addition. Studer demonstrated the reaction between electrophilic radicals generated from iodides (including perfluoroalkyl iodides) and the ate complexes generated in situ

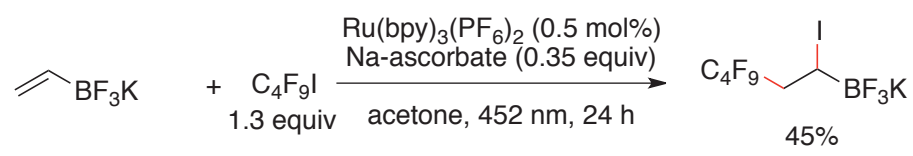

Scheme 4. Kublicki's iodine atom addition over vinylic trifluoroboronates.

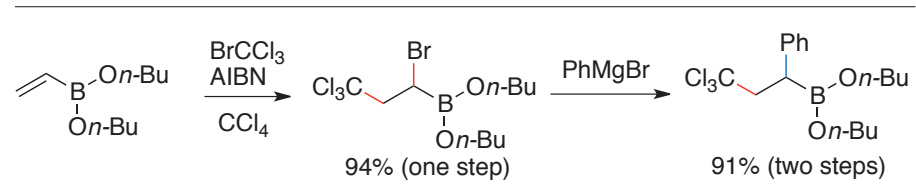

Scheme 5. Discovery of the 1,2-metallate rearrangement by Matteson and Mah (red bond: radical reaction; blue bond: ionic process (1,2-metallate rearrangement)).

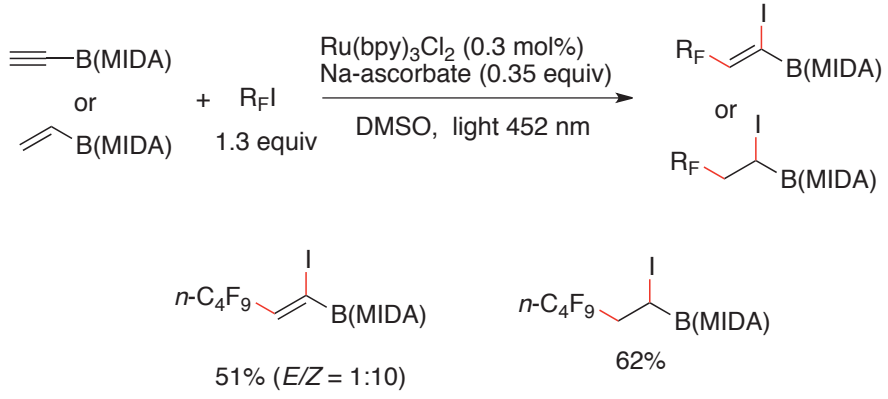

Scheme 3. Iodine atom transfer addition over MIDA vinyl- and acetynylboronates.

by treatment of pinacol vinylboronate with alkyl- and aryl-lithium derivatives (Scheme 6). ${ }^{[26]}$ The reaction was initiated with triethylborane and residual oxygen in the solvent, although later visible light was preferentially employed as initiator.[27]

Soon after, Aggarwal and co-workers reported a similar reaction initiated by light under photoredox catalysis (Scheme 7). ${ }^{28]}$ For many substrates, the reaction was found to work in the absence of the $\mathrm{Ru}$ photoredox catalyst demonstrating that a radical chain process was also operating.

Soon after these reports, Renaud and co-workers reported the same three-component coupling process with a large substrate scope initiated by triethylborane and di-tert-butylhyponitrite (DTBHN) (Scheme 8). [29] The reactions were performed in tertbutyl methyl ether (TBME) to minimize side products resulting from hydrogen atom transfer processes and to avoid the solvent switch/dilution procedures described by Studer $\left(\mathrm{Et}_{2} \mathrm{O}\right.$ to acetonitrile) and Aggarwal ( $\mathrm{Et}_{2} \mathrm{O}$ into 1,3-dimethyl-2-imidazolidinone (= DMI)). Issues of allylic deprotonation of the isopropenylboronic esters with tert-butyllithium were avoided by conducting the reactions at low temperature.

\section{Mechanistic Investigations}

Three different mechanistic pathways were suggested for this reaction and each challenged by experiment. ${ }^{[29]}$ In pathway 1 , an atom transfer radical addition (ATRA) reaction leading to an $\alpha$-haloboronate $\mathbf{I}$ that undergoes the classical [1,2]-metalate rearrangement was envisaged (Scheme 9). This mechanism was discarded based on a radical clock experiment. In a reaction with a prenylated bromomalonate, an atom transfer process should have led to the formation of a cyclopentane derivative since the 5-exotrig cyclization step is expected to be much faster than the bromine atom transfer step. However, the reaction afforded exclusively the acyclic three-component coupling product indicating that the

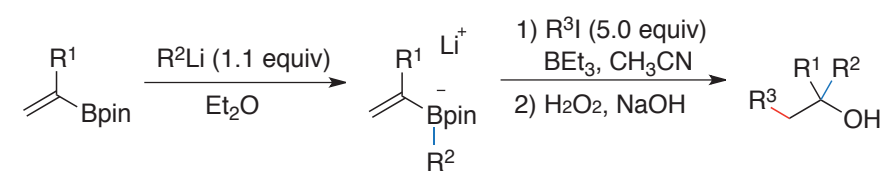

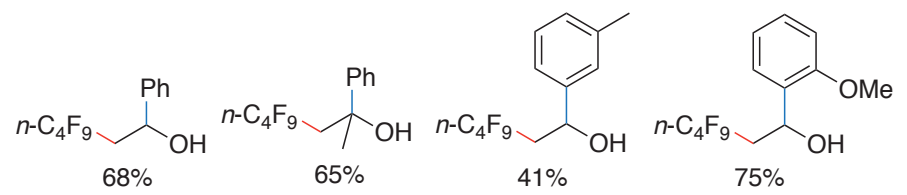

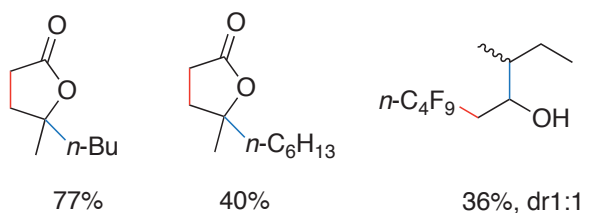

Scheme 6. Studer's three-component coupling reaction with alkenylboronates. 


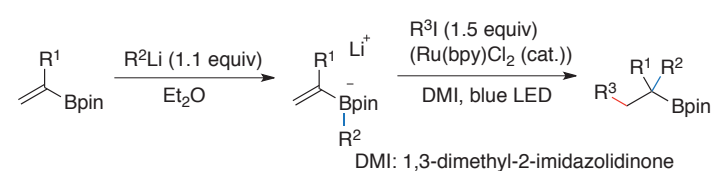$$
\begin{aligned}
& 88 \% \mathrm{R}^{2}=n-\mathrm{Bu} \\
& 88 \% \mathrm{R}^{2}=i-\mathrm{Pr} \\
& 74 \% \mathrm{R}^{2}=t-\mathrm{Bu}
\end{aligned}
$$

$$
\underbrace{\text { Bpin }}_{\substack{85 \% \\ \text { (Ru) }}}
$$

Scheme 7. Aggarwal's three-component coupling reaction with alkenylboronates.

1,2-shift was significantly faster than the cyclization and therefore could not involve an atom transfer process.

The second pathway that was examined was a radical [1,2]-alkyl or aryl migration leading to a boryl radical-anion II that could then propagate the chain via a reductive single electron transfer (SET) process. This mechanism could be discarded by running a cyclization reaction starting from a selenide under tin hydride conditions. Under these conditions, no product resulting from a 1,2-shift process could be detected (Scheme 10) indicating that a radical 1,2-shift process is much slower than the hydrogen atom transfer step involving $\mathrm{Bu}_{3} \mathrm{SnH}$ and therefore not compatible with the extremely fast 1,2-migration observed with the prenylated malonate in Scheme 9.

In the third considered pathway, the radical anion resulting from the radical addition to the vinylboronate reduces the starting halide via a single electron transfer (SET) affording the inverse ylid III which subsequently undergoes a fast 1,2-metallate rearrangement (Scheme 11). Performing the reaction with 2-bromoisobutyronitrile gave the expected three-component coupling product in $21 \%$ yield. However, running the reaction with the corresponding phenylselenide, which is less prone to SET reduction, gave no trace of the three-component coupling product. This experiment supports a SET mechanism when a
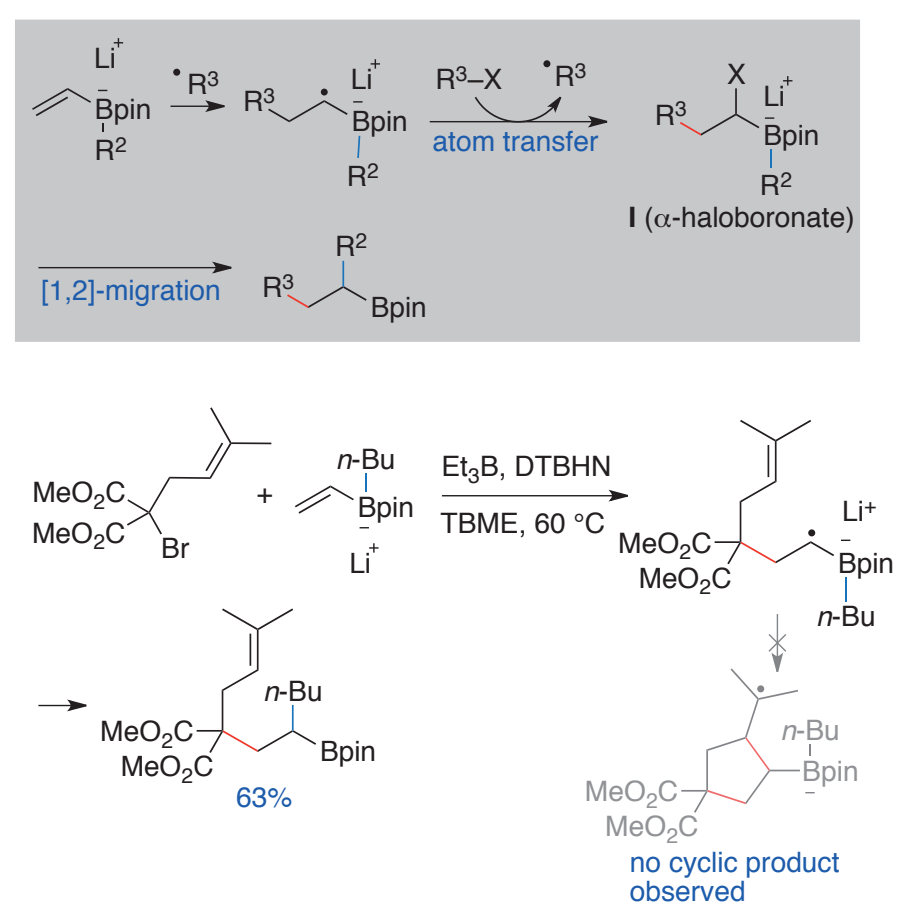

Scheme 9. Experiment to discard the ATRA mechanism.
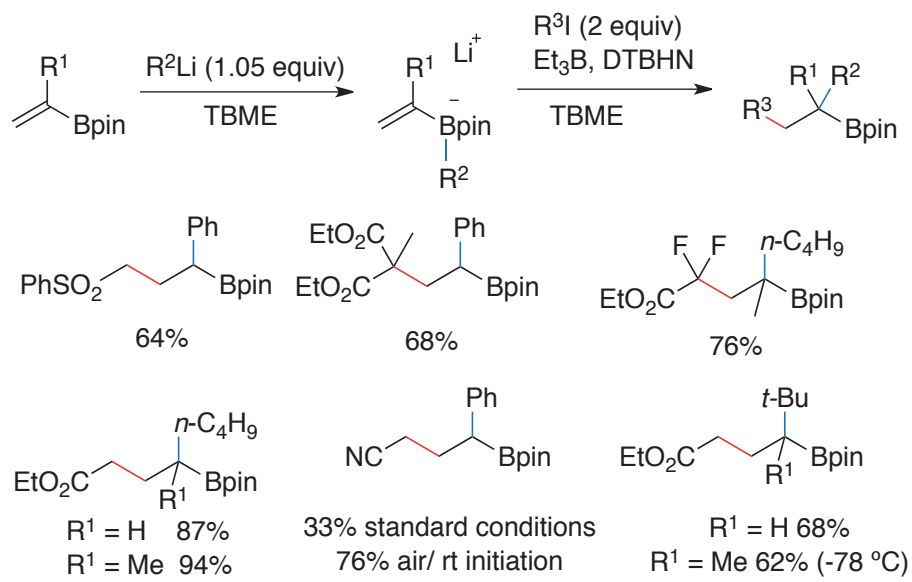

Scheme 8. Renaud's three-component coupling reaction with alkenylboronates.

bromide or an iodide is used as a radical precursor and allows to further disprove the ATRA mechanism presented in Scheme 9.

The groups of Studer and Aggarwal also suggested a SET mechanism was operating based on experimental results. For instance, Studer and co-workers reported the trifluoromethylation of the PhLi ate-complex with Togni's reagent (Scheme 12, A). ${ }^{[26]}$ Since this trifluoromethylating reagent is well-documented to operate though a radical mechanism (no $\left[\mathrm{CF}_{3}\right]^{+}$is generated at any point), ${ }^{[30]}$ this experiment is a compelling piece of evidence for the oxidation pathway. This result does not necessarily imply that iodide and bromide radical precursors also react via this pathway but computational investigation of the reaction with $\mathrm{CF}_{3} \mathrm{I}$ support a nearly barrierless SET. Aggarwal and co-workers provided more evidence of a radical-SET pathway in their trifluoromethylation reaction with Umemoto's reagent (see Scheme 15 below). ${ }^{[31]}$ They provided direct spectroscopic evidence of radical formation by intercepting the transient trifluoromethyl radical with the spin trap $N$-tert-butyl- $\alpha$-phenylnitrone (PBN) so that they could record the electron paramagnetic resonance (EPR) spectrum of the corresponding persistent nitroxide (Scheme 12).
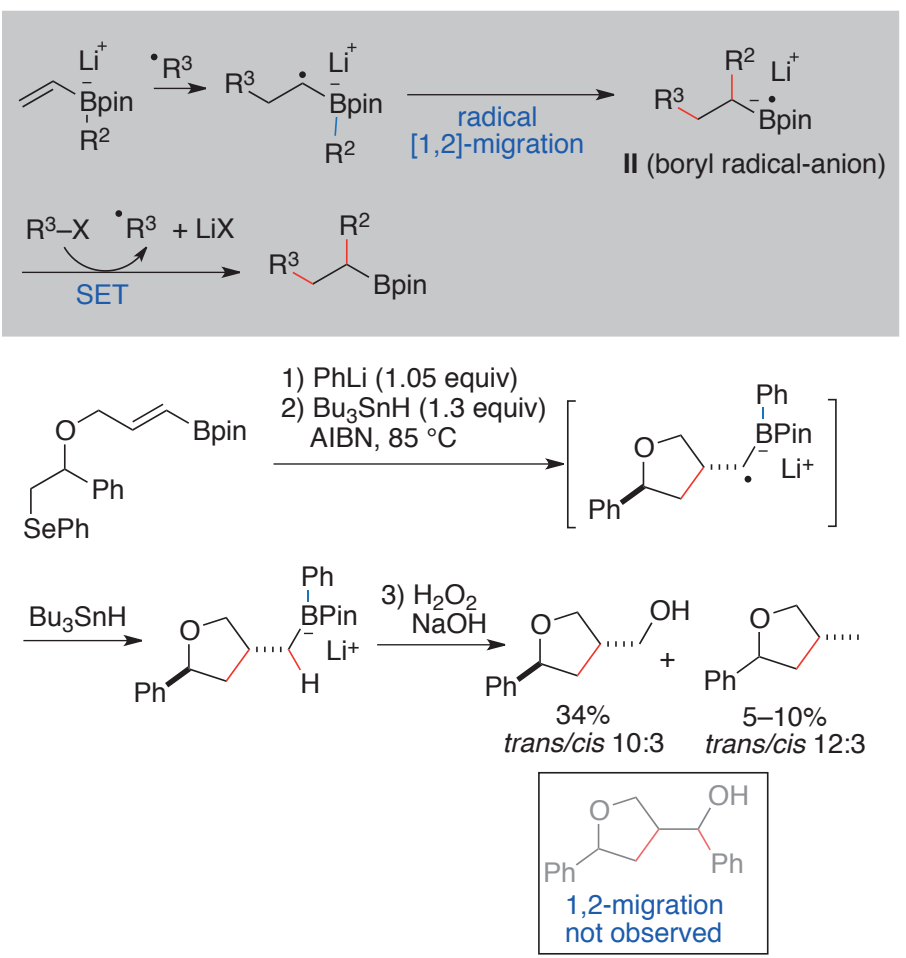

Scheme 10. Experiment to disprove the radical 1,2-shift. 


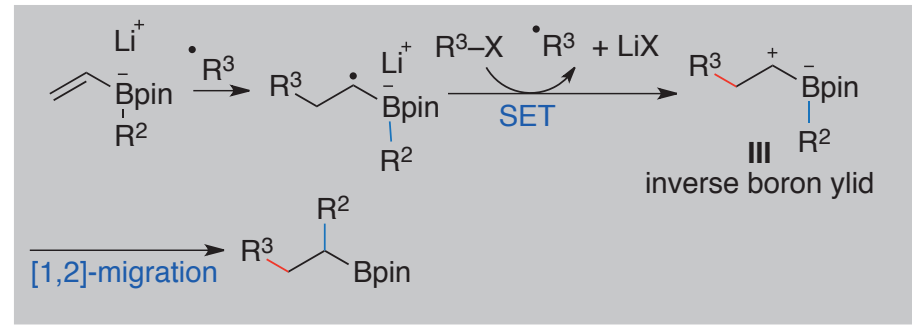

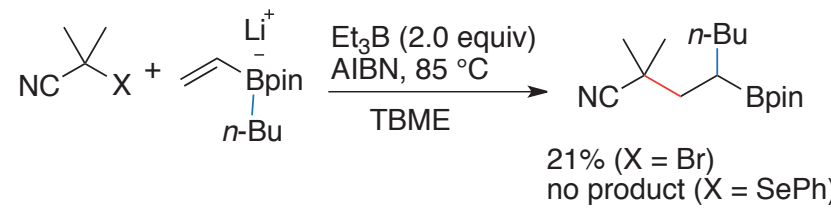

Scheme 11. Evidence for a single electron transfer mechanism.

\section{Further Synthetic Applications}

Studer has developed a method to access enantiomerically enriched $\alpha$-alkyl ketones from enantiomerically pure alkylboronic esters. ${ }^{[32]}$ In this approach, the alkenylboronate complexes were generated by adding vinyllithium to chiral pinacol alkylboronic esters. The absolute configuration at the migrating alkyl group was preserved during the process. However, the newly formed chiral center bearing the boronic residue was obtained as a mixture of diastereomers. After oxidative work-up, the diastereomeric mixture of alcohols was oxidized to the corresponding ketones. Alternatively, chiral alkanes were accessed after radical mediated protodeboronation involving treatment of the pinacol boronic ester with tetrabutylammonium fluoride (TBAF) and $\mathrm{Mn}$ (III) acetate in the presence of tert-butylcatechol (TBC) (Scheme 13).

Dienyl systems work in a similar fashion, simple light irradiation in the absence of any additive or photocatalyst was enough to initiate the reaction. ${ }^{[33]}$ This procedure allows the preparation of complex allylboranes with good control of the stereochemistry of the double bond (Scheme 14). Radical addition occurs with full regioselectivity for the terminus position of the dienyl moiety.

Aggarwal and co-workers were able to extend their reaction to the preparation of 2-trifluoromethylated 5-alkylated or 5-arylated fu-

$$
\begin{aligned}
& R_{R^{2}}^{\text {Bpin }} R^{3} \\
& \mathrm{R}_{\mathrm{R}^{2}}^{11} \mathrm{R}^{3} \quad 60 \% \text {, er > }>98: 2 \text { Et } \\
& \checkmark \mathrm{Li}_{\mathrm{Li}} \quad \mathrm{NaBO}_{\text {periodinane }} \text { Dess-Martin } \\
& \text { - } \mathrm{Li}^{+} t-\mathrm{BuCO}_{2} \mathrm{CH}_{2} \mathrm{I} \quad \mathrm{pinB} \quad \text { TBAF, TBC }
\end{aligned}
$$

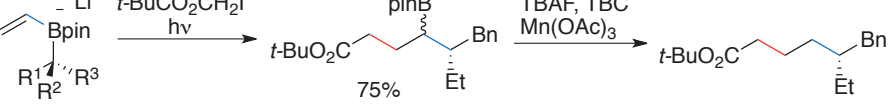

$$
\begin{aligned}
& 76 \% \text {, er }>99: 1 \\
& \text { 2) } \mathrm{H}_{2} \mathrm{O}_{2}, \mathrm{NaOH} \text { then } \\
& \stackrel{\text { Dess-Martin periodinane }}{\longrightarrow}
\end{aligned}
$$

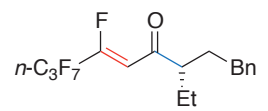

$$
\begin{aligned}
& 46 \% \text {, er }>99: 1
\end{aligned}
$$

Scheme 13. Studer's extension to access chiral ketones and alkanes.

$$
\text { (1) }
$$

Scheme 14. Studer's extension to generate allylboronic esters.

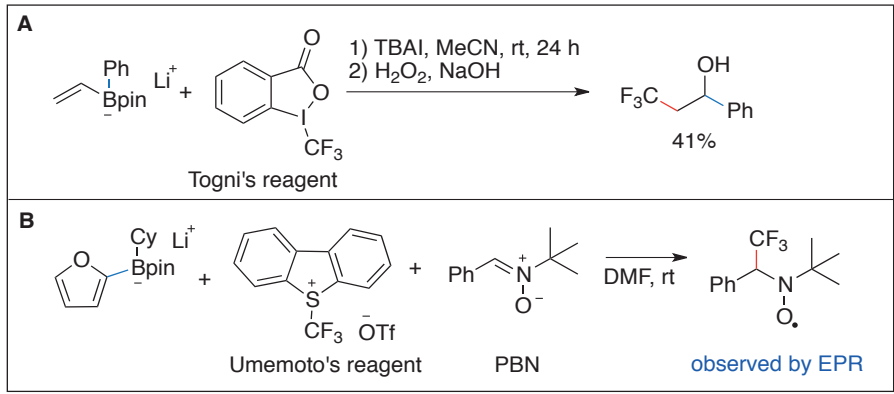

Scheme 12. Further evidence for a radical-polar crossover mechanism.

rans and pyrroles via trifluoromethyl radical addition to 2-furanyl/2pyranyl boronic esters followed by a rearomatization under oxidative conditions (Scheme 15). ${ }^{31]}$ The trifluoromethyl radical was generated by single electron reduction of Uemoto's reagent.

This reaction was extended to other sources of electrophilic radicals generated from the corresponding iodides. ${ }^{[34]}$ The use of a $\mathrm{Ru}$ (II) photoredox catalyst was required with furans (Scheme 16). The more nucleophilic indole derivatives underwent the same transformation via an ionic mechanism since they operated without any photoredox catalyst and in the presence of the radical inhibitor 1,1-diphenylethylene.

Shi has extended this chemistry to a 1,2-boryl migration leading to gem-diboronates derivatives. ${ }^{[35]}$ Alkenyl diboronate complexes in situ generated from alkenyl Grignard reagents and bis(pinacolato)diboron were reacted with alkyl bromides. Under ruthenium photoinitiation, gem-bis(boryl)alkanes were obtained in good yields (Scheme 17).

Studer and co-workers reported the selective $\alpha-\mathrm{C}-\mathrm{H}$ abstraction by in situ generated trifluoromethyl radicals from alkyl(aryl) boronate complexes prepared from aryl pinacol boronic esters and alkyl lithium reagents (Scheme 18). ${ }^{[36]}$ The transient radical anions
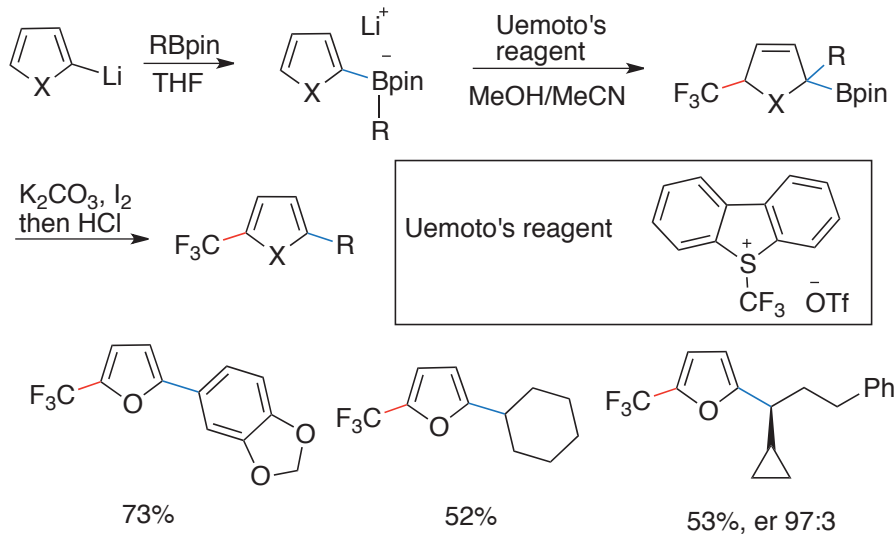

Scheme 15. Aggarwal's extension to synthesize trifluoromethylated furans and pyrroles.

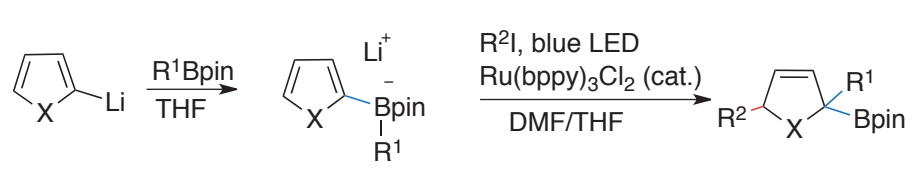$$
\stackrel{\mathrm{I}_{2}, \mathrm{KOAC}}{\longrightarrow} \mathrm{R}^{2}{ }_{\mathrm{X}}{ }_{\mathrm{R}^{1}}
$$

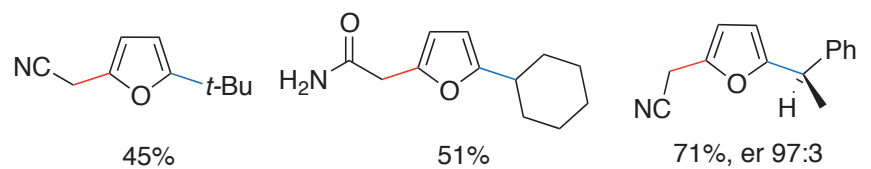

Scheme 16. Aggarwal's extension to synthesize alkylated furans and pyrroles. 


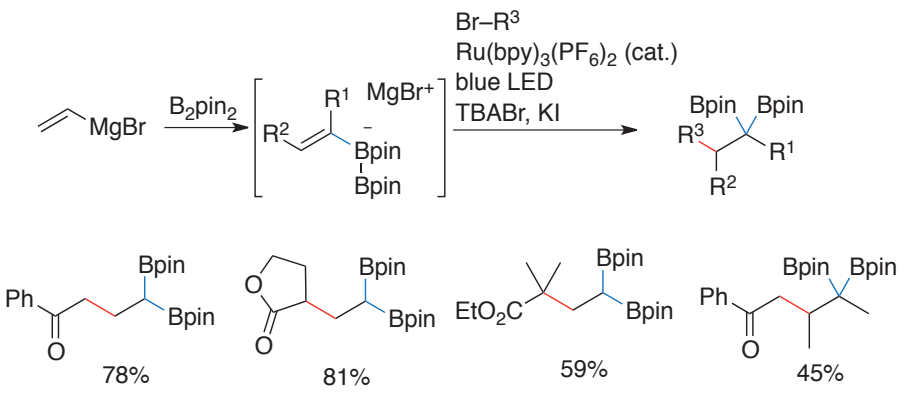

Scheme 17. Shi's extension to synthesize gem-bis(boryl)alkanes.

undergo electron transfer oxidation followed by 1,2-aryl/alkyl migration from boron to carbon to give $\alpha$-arylated boronic esters. In this process, $\mathrm{CF}_{3} \mathrm{I}$ is acting as an oxidant and a source of $\mathrm{CF}_{3} \bullet$ radicals involved in the hydrogen atom transfer (HAT). The regioselectivity of the HAT is remarkable and results from strong polar effects favoring abstraction at the $\alpha$-position of the boronate complex.

Another application of this type of chemistry is seen by the radical addition to bicyclobutylboronate complexes reported by Aggarwal (Scheme 19). ${ }^{[37]}$ The strain of the $\sigma$-bond creates a good radical acceptor with concomitant bond breakage to furnish borylated cyclobutanes with high diastereoselectivity. The resulting 1-boryl radical, after radical addition, transfers an electron as for the previous reactions to trigger the 1,2-shift. The stereochemical control is occurring during the SET step by minimizing steric interactions between $R^{2} I$ and the $R^{1 / R^{2}}$ groups of the radical anion.

\section{Outlook and Conclusions}

Control of the stereochemical outcome of the 1,2-migration process is problematic. More effort is still heavily needed since

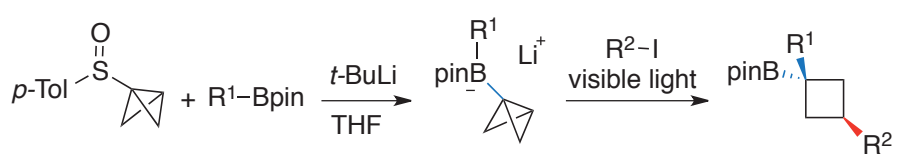

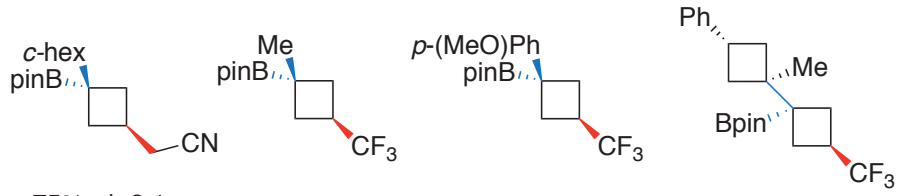

$75 \%$, dr 3:1 $\quad 66 \%$, dr $11: 1 \quad 62 \%$, dr 6.8:1 $\quad 41 \%$, dr $12.5: 1$

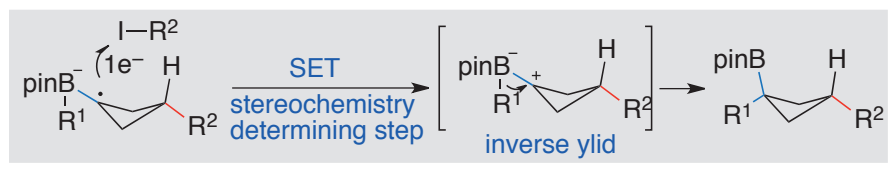

Scheme 19. Radical addition over $\sigma$-bond of bicyclobutylboronates.

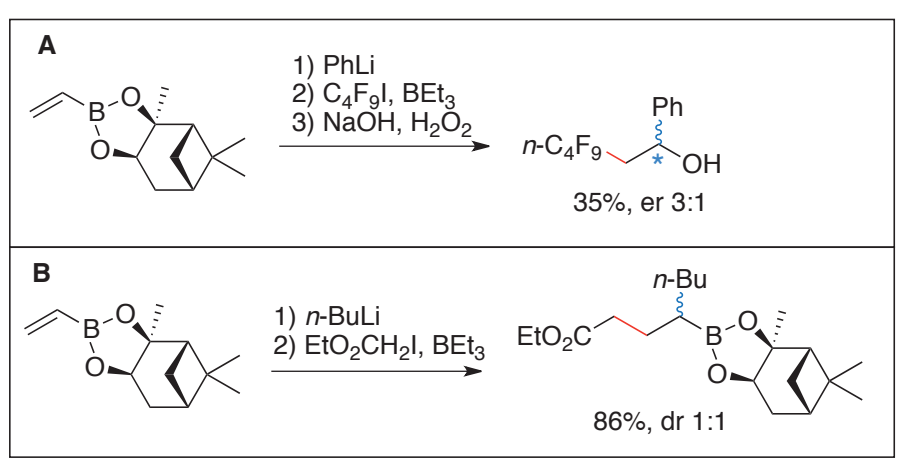

Scheme 20. Initial attempts by Studer (A) and Renaud (B) to control the stereochemistry of the 1,2-migration process.
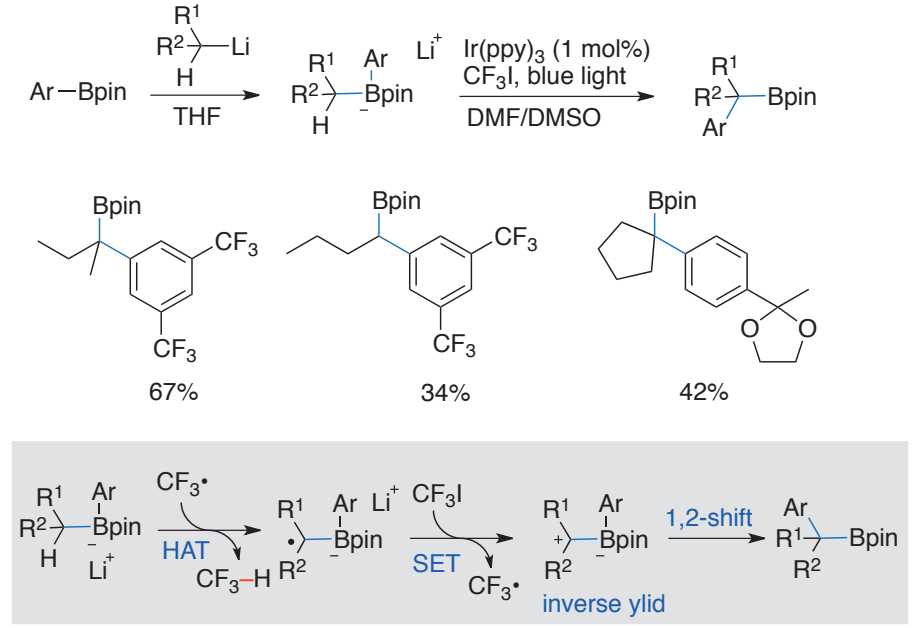

Scheme 18. Hydrogen atom transfer induced 1,2-metallate rearrangement.

preliminary attempts by Studer (Scheme 20, A) and Renaud (Scheme 20, B) of a chiral auxiliary control with pinanediol vinylboronic ester gave the three-component coupling products with low or no stereocontrol.

Very recently, Morken reported a closely related three-component coupling process involving a radical addition to pinacol vinyl boronic ester followed by a nickel-catalyzed cross-coupling process with organozinc reagents (Scheme 21). ${ }^{[38]}$ This alternative approach follows a fundamentally different mechanism and is nicely complementary to the chemistry described here since it works with nucleophilic radicals generated from tertiary alkyl iodides.

In conclusion, the three-component coupling reaction presented here opens new opportunities for the selective and highly convergent construction of functionalized and versatile building blocks. Applications in the field of synthesis of products of biological, medicinal, pharmaceutical, and material importance are expected.

\section{Acknowledgements}

The Swiss National Science Foundation (Project 200020_172621) and the University of Bern are gratefully acknowledged for financial support.

Received: December 16, 2019

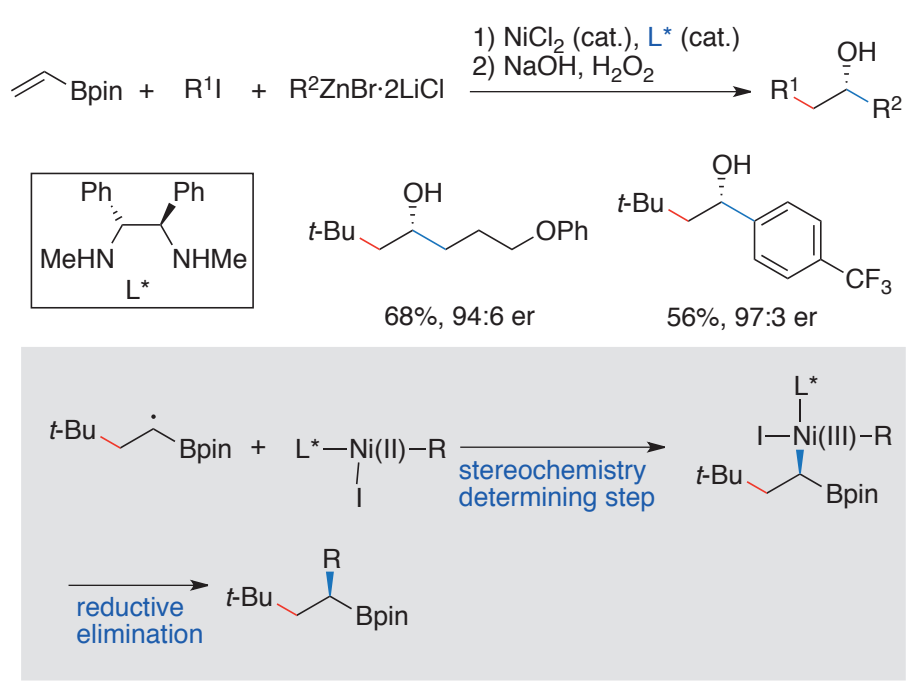

Scheme 21. Morken's approach to enantioselective three-component coupling reactions. 
[1] N. Miyaura, A. Suzuki, Chem. Rev. 1995, 95, 2457, DOI: 10.1021/ cr00039a007.

[2] N. Miyaura, in 'Topics in Current Chemistry: Cross-Coupling Reactions', Vol. 219, Ed. N. Miyaura, Springer Berlin Heidelberg, Berlin, Heidelberg, 2002, pp. 11, DOI: 10.1007/3-540-45313-X_2.

[3] C. Ollivier, P. Renaud, Chem. Rev. 2001, 101, 3415, DOI: 10.1021/ cr010001p.

[4] V. Darmency, P. Renaud, in 'Topics in Current Chemistry: Radicals in Synthesis I', Vol. 263, Ed. A. Gansäuer, Springer-Verlag, Berlin, Heidelberg, 2006, pp. 71, DOI: 10.1007/128_030.

[5] P. Renaud, A. Beauseigneur, A. Brecht-Forster, B. Becattini, V. Darmency, S. Kandhasamy, F. Montermini, C. Ollivier, P. Panchaud, D. Pozzi, E. M. Scanlan, A.-P. Schaffner, V. Weber, Pure Appl. Chem. 2007, 79, 223, DOI: 10.1351/pac200779020223.

[6] P. Renaud, in 'Encyclopedia of Radicals in Chemistry, Biology and Materials', Vol. 2 (Synthetic Strategies and Applications), Eds. C. Chatgilialoglu, A. Studer, Wiley, Chichester, UK, 2012, DOI: 10.1002/9780470971253. $\operatorname{rad} 020$.

[7] N. Guennouni, F. Lhermitte, S. Cochard, B. Carboni, Tetrahedron 1995, 51, 6999, DOI: 10.1016/0040-4020(95)00358-F.

[8] A. Noble, R. S. Mega, D. Pflästerer, E. L. Myers, V. K. Aggarwal, Angew. Chem. Int. Ed. 2018, 57, 2155, DOI: 10.1002/anie.201712186.

[9] B. Quiclet-Sire, S. Z. Zard, J. Am. Chem. Soc. 2015, 137, 6762, DOI: 10.1021/jacs.5b03893.

[10] S. Namirembe, J. P. Morken, Chem. Soc. Rev. 2019, 48, 3464, DOI: 10.1039/ $\mathrm{C} 9 \mathrm{CS} 00180 \mathrm{H}$.

[11] A. McCarroll, J. C. Walton, A. McCarroll, R. Nziengui, B. Carboni, Chem. Commun. 1997, 2075, DOI: 10.1039/a703950f.

[12] J. C. Walton, A. J. McCarroll, Q. Chen, B. Carboni, R. Nziengui, J. Am. Chem. Soc. 2000, 122, 5455, DOI: 10.1021/ja9944812.

[13] H. Lopez-Ruiz, S. Z. Zard, Chem. Commun. 2001, 2618, DOI: 10.1039/ b109637k.

[14] M. R. Heinrich, L. A. Sharp, S. Z. Zard, Chem. Commun. 2005, 3077, DOI: $10.1039 / \mathrm{b} 502885 \mathrm{j}$.

[15] M. Kublicki, M. Dąbrowski, K. Durka, T. Kliś, J. Serwatowski, K. Woźniak, Tetrahedron Lett. 2017, 58, 2162, DOI: 10.1016/j.tetlet.2017.04.075.

[16] M. Kublicki, K. Durka, T. Kliś, Tetrahedron Lett. 2018, 59, 2700, DOI: 10.1016/j.tetlet.2018.05.086.

[17] H. Kim, D. W. C. MacMillan, J. Am. Chem. Soc. 2008, 130, 398, DOI: 10.1021/ja077212h.

[18] Y. Yasu, T. Koike, M. Akita, Chem. Commun. 2013, 49, 2037, DOI: 10.1039/ C3CC39235J.

[19] T. Koike, M. Akita, Acc. Chem. Res. 2016, 49, 1937, DOI: 10.1021/acs. accounts.6b00268.

[20] D. Fernandez Reina, A. Ruffoni, Y. S. S. Al-Faiyz, J. J. Douglas, N. S. Sheikh, D. Leonori, ACS Catal. 2017, 7, 4126, DOI: 10.1021/acscatal.7b01120.

[21] D. S. Matteson, J. Am. Chem. Soc. 1959, 81, 5004, DOI: 10.1021/ ja01527a068.
[22] D. S. Matteson, J. Am. Chem. Soc. 1960, 82, 4228, DOI: 10.1021/ ja01501a029.

[23] D. S. Matteson, R. W. H. Mah, J. Am. Chem. Soc. 1963, 85, 2599, DOI: 10.1021/ja00900a017.

[24] D. S. Matteson, J. Org. Chem. 2013, 78, 10009, DOI: 10.1021/jo4013942.

[25] D. S. Matteson, in 'Boron Reagents in Synthesis', Vol. 1236, American Chemical Society, 2016, pp. 173, DOI: 10.1021/bk-2016-1236.ch005.

[26] M. Kischkewitz, K. Okamoto, C. Mück-Lichtenfeld, A. Studer, Science 2017, 355, 936, DOI: $10.1126 /$ science.aal3803.

[27] M. Kischkewitz, A. Studer, Org. Synth. 2018, 95, 205, DOI: 10.15227/orgsyn.095.0205.

[28] M. Silvi, C. Sandford, V. K. Aggarwal, J. Am. Chem. Soc. 2017, 139, 5736, DOI: $10.1021 /$ jacs.7b02569.

[29] N. D. C. Tappin, M. Gnägi-Lux, P. Renaud, Chem. Eur. J. 2018, 24, 11498, DOI: $10.1002 /$ chem.201802384.

[30] J. Charpentier, N. Früh, A. Togni, Chem. Rev. 2015, 115, 650, DOI: 10.1021/ cr500223h.

[31] Y. Wang, A. Noble, C. Sandford, V. K. Aggarwal, Angew. Chem. Int. Ed. 2017, 56, 1810, DOI: 10.1002/anie.201611058.

[32] C. Gerleve, M. Kischkewitz, A. Studer, Angew. Chem. Int. Ed. 2018, 57, 2441, DOI: 10.1002/anie.201711390.

[33] M. Kischkewitz, C. Gerleve, A. Studer, Org. Lett. 2018, 20, 3666, DOI: 10.1021/acs.orglett.8b01459.

[34] M. Silvi, R. Schrof, A. Noble, V. K. Aggarwal, Chem. Eur. J. 2018, 24, 4279, DOI: $10.1002 /$ chem.201800527.

[35] B. Zhao, Z. Li, Y. Wu, Y. Wang, J. Qian, Y. Yuan, Z. Shi, Angew. Chem. Int. Ed. 2019, 58, 9448, DOI: 10.1002/anie.201903721.

[36] D. Wang, C. Mück-Lichtenfeld, A. Studer, J. Am. Chem. Soc. 2019, 141, 14126, DOI: 10.1021/jacs.9b07960.

[37] M. Silvi, V. K. Aggarwal, J. Am. Chem. Soc. 2019, 141, 9511, DOI: 10.1021/ jacs.9b03653.

[38] M. Chierchia, P. Xu, G. J. Lovinger, J. P. Morken, Angew. Chem. Int. Ed. 2019, 58, 14245, DOI: 10.1002/anie.201908029.

\section{License and Terms}

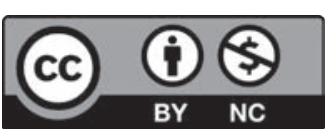

This is an Open Access article under the terms of the Creative Commons Attribution License CC BY_NC 4.0. The material may not be used for commercial purposes.

The license is subject to the CHIMIA terms and conditions: (http:// chimia.ch/component/sppagebuilder/?view=page\&id=12).

The definitive version of this article is the electronic one that can be found at doi:10.2533/chimia.2020.33 